\title{
Tumor mutation burden in lung cancer: a new predictive biomarker for immunotherapy or too soon to tell?
}

\author{
Mariam Alexander, Jose Galeas, Haiying Cheng \\ Department of Oncology, Montefiore Medical Center/Albert Einstein College of Medicine, Bronx, NY, USA \\ Correspondence to: Haiying Cheng, MD, PhD. Department of Oncology, Montefiore Medical Center/Albert Einstein College of Medicine, Bronx, \\ NY10461, USA. Email: hcheng@montefiore.org. \\ Provenance: This is an invited Editorial commissioned by the Section Editor Dr. Lei Deng (Department of Medicine, Jacobi Medical Center, Albert \\ Einstein College of Medicine, Bronx, NY, USA). \\ Comment on: Hellmann MD, Ciuleanu TE, Pluzanski A, et al. Nivolumab plus Ipilimumab in Lung Cancer with a High Tumor Mutational Burden. \\ N Engl J Med 2018;378:2093-104.
}

Submitted Aug 30, 2018. Accepted for publication Sep 07, 2018.

doi: $10.21037 /$ jtd.2018.09.35

View this article at: http://dx.doi.org/10.21037/jtd.2018.09.35

Immunotherapy with immune-checkpoint inhibitors (ICIs) has shifted the therapeutic landscape in lung cancer. At the same time, the clinical benefits of ICIs in unselected patients remain modest. Thus, there is a growing need to identify predictive biomarkers for better patient selection. So far, only PD-L1 expression (1) and microsatellite instability (MSI) (2) are associated with Food and Drug Administration (FDA)-approved indications for ICIs. In the front-line setting, the pivotal KEYNOTE-024 trial revealed that the PD-1 antibody, pembrolizumab, was better than chemotherapy in patients with advanced non-small cell lung cancer (NSCLC) and high PD-L1 expression [Tumor Proportion Score (TPS) $\geq 50 \%$ ] (1). Pembrolizumab led to significantly longer progression free survival (PFS) and overall survival (OS) (1) in this patient subset. The more recent KEYNOTE-042 study further reported that pembrolizumab was superior to chemotherapy in patients with PD-L1 TPS $\geq 1 \%$ with more clinical efficacy and less side effects; Additionally, the OS benefits was greater in patients with higher PD-L1 (TPS $\geq 50 \%$ ), which was the main driver of the benefits observed in the trial (3). The combined activity of pembrolizumab and chemotherapy was assessed in the KEYNOTE-189 study (4). In patients with advanced non-squamous NSCLC and no active EGFR/ $A L K$ alterations, the combination resulted in significantly improved PFS and OS than chemotherapy, irrespective of PD-L1 expression. The greatest benefit was seen in patients with PD-L1 score of $\geq 50 \%$ [hazard ratio (HR) 0.42 ], although patients with a PD-L1 expression $<1 \%$ also benefited (HR 0.59) (4). These data also implicate that, as a biomarker, PD-L1 expression alone is not flawless to predict which patient group will benefit from immunotherapy. Despite being imperfect, PD-L1 expression is a biomarker commonly used in NSCLC to identify patients for ICIs, especially at the front-line setting as monotherapy.

There are other emerging predictors for response or resistance to immunotherapy, including tumor mutation burden (TMB). TMB can be defined as the total number of nonsynonymous mutations present in a tumor (5). High mutation load correlates with an immunogenic tumor microenvironment with increased expression of tumor specific neo-antigens that can be targeted by activated immune cells $(6,7)$. The main pathways contributing to mutation rate include the DNA damage repair and DNA replication pathways. Inactivation of the DNA mismatch repair (dMMR) pathway, which is critical to maintain fidelity of replication, has been shown to correlate with a high TMB in tumors (8) and are more likely to benefit from immunotherapy (2). Mutations in DNA replication genes, such as POLD1 and POLE, are also associated with increased mutation frequency and high TMB (5). TMB are usually measured with whole exome sequencing (WES) or comprehensive genomic profiling (CGP, or gene panels sequencing). WES is not widely available for routine clinical practice due to its high cost and long turn-around time. CGP is more commonly performed to 
check TMB and genetic alterations. FoundationOne CDx and MSK-IMPACT tumor profiling are two FDA-approved CGP assays to characterize individual's genomic profiles, including TMB status.

Hellmann et al. recently reported a part of data from the phase III CheckMate227 study: the analysis suggest that TMB may be a useful biomarker to predict response to the combination therapy of ipilimumab (CTLA-4 inhibitor) plus nivolumab (PD-1 inhibitor) in the first line setting of stage IV or recurrent NSCLC (9). TMB was determined by the FoundationOne CDx assay. For this specific part of the CheckMate227 trial, the high TMB was defined as at least 10 mutations per megabase $(\geq 10 \mathrm{Mut} / \mathrm{Mb})$. Among patients with high TMB ( $\geq 10 \mathrm{Mut} / \mathrm{Mb})$, the 1 -year PFS rate was significantly higher for nivolumab plus ipilimumab vs. chemotherapy (42.6\% vs. 13.2\%), and the median PFS was longer (7.2 vs. 5.5 months; HR 0.58; $\mathrm{P}<0.001)$. The response was more durable for the combined ICIs: $68 \%$ had ongoing response at 1 year with nivolumab plus ipilimumab, compared to $25 \%$ with chemotherapy. Furthermore, the benefit of nivolumab plus ipilimumab over chemotherapy in the TMB high patients was independent of the PD-L1 expression. No superior benefit of nivolumab plus ipilimumab to chemotherapy was observed in the TMB low group ( $<10 \mathrm{Mut} / \mathrm{Mb}$ ). The grade 3 or 4 toxicities were similar between two groups (Nivo + Ipi: $31.2 \%$ vs. chemo: $36.1 \%)$. Of note, the TMB-high cut off was different in the nivolumab monotherapy group ( $>13$ mutations/Mb) and there was no difference in PFS between the nivolumab monotherapy group compared to the chemotherapy. The authors thereby propose that nivolumab plus ipilimumab may stand for a front-line regimen in patients with high TMB, regardless of PD-L1 expression.

The modification of CheckMate 227 trial and the selection of its TMB thresholds were based on earlier studies (Table 1). Rizvi et al. performed WES analysis of two independent NSCLC cohorts $(\mathrm{n}=16$ for the discovery and $\mathrm{n}=18$ for the validation cohort) (10). They found that the higher nonsynonymous TMB was associated with better clinical efficacy of pembrolizumab, with superior response rate, more durable benefit and longer PFS. The median number of nonsynonymous mutations in patients with durable benefits $v s$. no durable benefits was $302 v s .148$ in the discovery group, and 244 vs. 125 in the validation cohort, respectively. Using the cut-off of nonsynonymous TMB $\geq 178$ resulted in the combined best sensitivity and specificity to predict pembrolizumab activity. The study also suggested a positive association between TMB and neoantigen burden, as well as DNA repair gene mutations.

In the phase I CheckMate012, the first-line nivolumab plus ipilimumab showed clinical activity and manageable toxicity in patients with advanced NSCLC (17). WES on tumor tissue and paired blood were performed on 75 patients from this study. Compared to those with low TMB ( $\leq$ median), patients with high TMB (> median, 158 mutations) were found to have better response, durable benefits and superior PFS (12). Independent of PD-L1 expression, TMB was the strongest predictor to the benefits of combined nivolumab and ipilimumab.

In the phase III CheckMate026, patients with untreated advanced NSCLC and PD-L1 expression $\geq 1 \%$ were assigned to receive nivolumab vs. chemotherapy (18). Exploratory analysis was conducted to investigate the relationship between clinical benefits and TMB (using WES). Patients were grouped based on TMB tertile distribution. In patients with high TMB ( $>243$ mutations), nivolumab was associated with higher response rate as compared to chemotherapy ( $47 \%$ vs. $28 \%$ ), and improved PFS (9.7 vs. 5.8 months, HR 0.62) (11). CheckMate026 also revealed lack of correlation between TMB and PD-L1 expression. On the other hand, patients with both high TMB and high PD-L1 had a higher response rate $(75 \%)$ compared to those with only one of these factors or neither factors.

Further evidence came from the phase II CheckMate568 study enrolling 288 patients with chemotherapy-naïve stage IV NSCLC, where TMB was found to be an independent biomarker of response to dual ICIs (nivolumab + ipilimumab), regardless of PD-L1 expression (13). TMB was assessed using the FoundationOne CDx assay. The objective response rate (ORR) was associated with TMB: the ORR was $4 \%, 10 \%, 44 \%$ and $39 \%$ in patients with $\mathrm{TMB}<5,<10, \geq 10$, and $\geq 15$ mutations/Mb. Subsequently, the TMB cut-off $\geq 10$ mutations/Mb was used in the phase III CheckMate 227 as aforementioned.

In a retrospective study of 240 patients with advanced NSCLC who received ICIs, TMB was mainly analyzed by the MSK-IMPACT sequencing (15). In a subset of patients $(n=49)$, TMB was assessed by both WES and MSK-IMPACT. There was a high correlation between the two assays $(\mathrm{P}=0.86 ; \mathrm{P}<0.001, \mathrm{n}=49)$. Median $\mathrm{TMB}$ was 7.4 single nucleotide variants (SNP)/Mb. TMB was higher in patients with complete/partial response $v s$. stable disease $v s$. progression of disease (median, 8.5 vs. 6.6 vs. $6.6 \mathrm{SNVs} /$ $\mathrm{Mb} ; \mathrm{P}=0.151)$. Moreover, patients with high TMB (>50\%) had longer PFS in comparison to patients with low TMB 


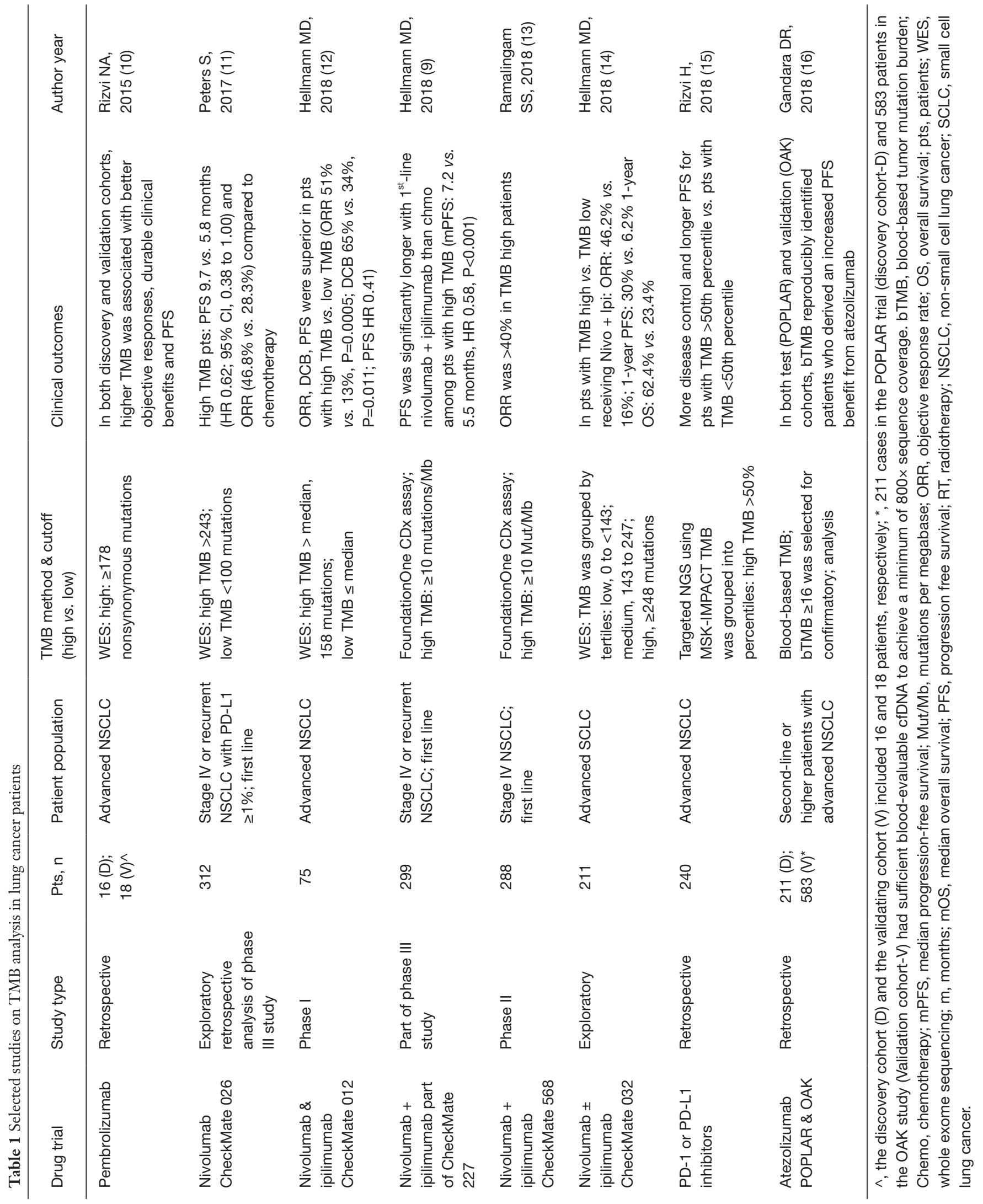


(<50\%). Consistent with previous studies, PD-L1 expression did not correlate with TMB $(\mathrm{P}=0.1915 ; \mathrm{n}=84)$. Either PD-L1 or TMB could independently predict clinical benefit with ICIs, whereas the composite of both (high TMB and PD-L1 > 1\%) further enriched the patient population who derived benefits from ICIs.

The predictive role of TMB to ICIs has also been investigated in advanced small cell lung cancer (SCLC) (CheckMate 032) (14) through WES. TMB was grouped by tertiles: low, 0 to $<143$ mutations; medium, 143 to 247 mutations; high, $\geq 248$ mutations. Both nivolumab monotherapy and combined nivolumab plus ipilimumab were associated with improved clinical efficacy in patients with high TMB, as compared to patients with low TMB. In particular, the ORR was as impressive as $46 \%$ for the dual ICIs in TMB high patients, along with prolonged 1-year PFS and 1-year OS. Thus, TMB may also be used as a biomarker to identify SCLC patients for ICIs.

In addition to tumor tissue biomarkers, blood-based assays are also being developed to check TMB in plasma (bTMB) and other circulating biomarkers. In a retrospective analysis using POPLAR trial as a test cohort while OAK study as a validation cohort, the bTMB measurement with hybridization-capture methodology (similar to FoundationOne), was found to be feasible and well-correlated with tissue TMB (16). Using cut-point of $\geq 16$ (prevalence of $27-30 \%$ in both cohorts), bTMB appeared to be a reproducible predictor to identify patients who derived improved PFS from atezolizumab therapy (16). The ongoing BFAST trial further investigates this bTMB assay in the front-line setting prospectively.

In summary, several lines of evidence indicate the potential roles of TMB in predicating clinical benefits to ICIs. However, similar to PD-L1 expression, TMB is also an imperfect biomarker requiring refinement. For instance, there are different assays, platforms and cut-offs to characterize TMB. None of them are FDA approved yet, due to lack of standardization and validation. Moreover, the OS data from Checkmate 227 and other studies are still eagerly awaited, which will shed lights on the true predicative value of TMB. Moreover, there are certain technical caveats with TMB analysis: (I) long turnaround time: tissue TMB analysis takes 2-3 weeks; (II) requirement of significant amount of tissue for analysis (19): for instance, only $58 \%$ of patients enrolled in Checkmate-227 had enough tissue available for TMB testing (9); (III) storage time: the mean mutation number decreased as sample storage time increased (20). Taken together, the recent promising results on using $\mathrm{TMB}$ as a predictor to immunotherapy are not practice-changing yet. Future studies are warranted to improve the standardization, shorten the turnaround time, and validate the diagnostic assays.

In current immunotherapy landscape, how can potential biomarkers guide us to choose ICIs in the first-line setting for patients with advanced NSCLC and no targetable alterations? TMB and tumor PD-L1 expression are independent biomarkers and are not correlated with each other $(9,11,16,18)$. At the same time, the complementary utilization of both appears to predict immunotherapy activity better than each alone (15). Thus, in light of CheckMate 227, KEYNOTE-024, KEYNOTE-189, IMpower150 and other exciting studies, we propose the following front-line strategies for patients with stage IV or recurrent NSCLC (no driver alterations): (I) PD-L1 TPS $\geq 50$ : pembrolizumab monotherapy (or combined ICI + platinum-based chemotherapy if high disease volume and reasonable patient condition; or nivolumab + ipilimumab if TMB $\geq 10$ Mut/Mb); (II) PD-L1 $<50 \%$ and TMB $\geq 10$ Mut/ $\mathrm{Mb}$, nivolumab + ipilimumab, or ICI + chemotherapy; (III) PD-L $1<50 \%$ and TMB $<10$ Mut/Mb: ICI + chemotherapy or chemotherapy. Nevertheless, this is a time filled with opportunities and challenges for the development of biomarkers to advance precision immunotherapy.

\section{Acknowledgements}

None.

\section{Footnote}

Conflicts of Interest: The authors have no conflicts of interest to declare.

\section{References}

1. Reck M, Rodriguez-Abreu D, Robinson AG, et al. Pembrolizumab versus Chemotherapy for PD-L1Positive Non-Small-Cell Lung Cancer. N Engl J Med 2016;375:1823-33.

2. Le DT, Uram JN, Wang H, et al. PD-1 Blockade in Tumors with Mismatch-Repair Deficiency. N Engl J Med 2015;372:2509-20.

3. Lopes G, Wu YL, Kudaba I, et al. Pembrolizumab (pembro) versus platinum-based chemotherapy (chemo) as first-line therapy for advanced/metastatic NSCLC with a 
PD-L1 tumor proportion score (TPS) $\geq 1 \%$ : Open-label, phase 3 KEYNOTE-042 study. J Clin Oncol 2018:36:abstr LBA4.

4. Gandhi L, Rodriguez-Abreu D, Gadgeel S, et al. Pembrolizumab plus Chemotherapy in Metastatic NonSmall-Cell Lung Cancer. N Engl J Med 2018;378:2078-92.

5. Chalmers ZR, Connelly CF, Fabrizio D, et al. Analysis of 100,000 human cancer genomes reveals the landscape of tumor mutational burden. Genome Med 2017;9:34.

6. Kim JM, Chen DS. Immune escape to PD-L1/PD-1 blockade: seven steps to success (or failure). Ann Oncol 2016;27:1492-504.

7. Schumacher TN, Schreiber RD. Neoantigens in cancer immunotherapy. Science 2015;348:69-74.

8. Peltomaki P. Role of DNA mismatch repair defects in the pathogenesis of human cancer. J Clin Oncol 2003;21:1174-9.

9. Hellmann MD, Ciuleanu TE, Pluzanski A, et al. Nivolumab plus Ipilimumab in Lung Cancer with a High Tumor Mutational Burden. N Engl J Med 2018;378:2093-104.

10. Rizvi NA, Hellmann MD, Snyder A, et al. Cancer immunology. Mutational landscape determines sensitivity to PD-1 blockade in non-small cell lung cancer. Science 2015;348:124-8.

11. Peters S, Creelan BD, Hellmann M, et al. Abstract CT082: Impact of tumor mutation burden on the efficacy of first-line nivolumab in stage iv or recurrent non-small cell lung cancer: An exploratory analysis of CheckMate 026. Washington, DC: Cancer Res 2017.

12. Hellmann MD, Nathanson T, Rizvi H, et al. Genomic Features of Response to Combination Immunotherapy in Patients with Advanced Non-Small-Cell Lung Cancer. Cancer Cell 2018;33:843-52.e4.

13. Ramalingam SS, Hellmann MD, Awad MM, et al. Tumor mutational burden (TMB) as a biomarker for clinical benefit from dual immune checkpoint blockade with nivolumab (nivo) + ipilimumab (ipi) in first-line (1L) nonsmall cell lung cancer (NSCLC): identification of TMB cutoff from CheckMate 568. Cancer Res 2018;78. doi: 10.1158/1538-7445.AM2018-CT078.

14. Hellmann MD, Callahan MK, Awad MM, et al. Tumor Mutational Burden and Efficacy of Nivolumab Monotherapy and in Combination with Ipilimumab in Small-Cell Lung Cancer. Cancer Cell 2018;33:853-61.e4.

15. Rizvi H, Sanchez-Vega F, La K, et al. Molecular Determinants of Response to Anti-Programmed Cell Death (PD)-1 and Anti-Programmed Death-Ligand 1 (PD-L1) Blockade in Patients With Non-Small-Cell Lung Cancer Profiled With Targeted Next-Generation Sequencing. J Clin Oncol 2018;36:633-41.

16. Gandara DR, Paul SM, Kowanetz M, et al. Bloodbased tumor mutational burden as a predictor of clinical benefit in non-small-cell lung cancer patients treated with atezolizumab. Nat Med 2018;24:1441-8.

17. Hellmann MD, Rizvi NA, Goldman JW, et al. Nivolumab plus ipilimumab as first-line treatment for advanced nonsmall-cell lung cancer (CheckMate 012): results of an open-label, phase 1, multicohort study. Lancet Oncol 2017;18:31-41.

18. Carbone DP, Reck M, Paz-Ares L, et al. First-Line Nivolumab in Stage IV or Recurrent Non-Small-Cell Lung Cancer. N Engl J Med 2017;376:2415-26.

19. Available online: https://www.foundationmedicine.com/ genomic-testing/foundation-one

20. Chen S, Liu M, Zhou Y, et al. The impact of sample storage time on calculating tumor mutation burden (TMB). J Clin Oncol 2018;36:abstr e24022.
Cite this article as: Alexander M, Galeas J, Cheng H. Tumor mutation burden in lung cancer: a new predictive biomarker for immunotherapy or too soon to tell? J Thorac Dis 2018;10(Suppl 33):S3994-S3998. doi: 10.21037/jtd.2018.09.35 